\title{
An Analysis of 3-Year Outcomes Following Canaloplasty for the Treatment of Open-Angle Glaucoma
}

\author{
Mahmoud A. Khaimi, ${ }^{1}$ Justin D. Dvorak, ${ }^{2}$ and Kai Ding ${ }^{2}$ \\ ${ }^{1}$ Dean McGee Eye Institute, University of Oklahoma Health Sciences Center, Oklahoma City, OK, USA \\ ${ }^{2}$ Department of Biostatistics and Epidemiology, University of Oklahoma Health Sciences Center, Oklahoma City, OK, USA
}

Correspondence should be addressed to Mahmoud A. Khaimi; mahmoud-khaimi@dmei.org

Received 3 April 2017; Revised 2 August 2017; Accepted 16 August 2017; Published 29 September 2017

Academic Editor: Vicente Zanon-Moreno

Copyright (C) 2017 Mahmoud A. Khaimi et al. This is an open access article distributed under the Creative Commons Attribution License, which permits unrestricted use, distribution, and reproduction in any medium, provided the original work is properly cited.

\begin{abstract}
Purpose. To report 3-year results investigating the safety and efficacy of canaloplasty for open-angle glaucoma. Setting. University of Oklahoma, Dean McGee Eye Institute, Oklahoma, United States of America. Design. Nonrandomized single-center retrospective chart review. Methods. Adult open-angle glaucoma eyes underwent canaloplasty or combined cataract-canaloplasty surgery. A tensioning suture was placed into Schlemm's canal in all eyes. Primary endpoints included the mean IOP and mean number of glaucoma medications at each follow-up visit. Secondary endpoints included visual acuity and surgical/postsurgical complications. Results. The study cohort included 277 eyes (mean age, 72.8 years). Overall, the mean baseline IOP of $19.7 \mathrm{mmHg}$ was reduced to $14.3 \mathrm{mmHg}, 14.0 \mathrm{mmHg}$, and $15.2 \mathrm{mmHg}$ at 1,2 , and 3 years, respectively $(p<0.001)$. The average medicine use was reduced from 2.1 preoperatively to 0.4 at 12 months, and 0.5 and 0.6 at two and three years, respectively $(p<0.001)$. The frequency of surgical and postsurgical complications was low with no serious adverse events recorded. Conclusion. Canaloplasty was safe and effective in achieving long-term IOP reductions and reduced dependence on antiglaucoma medications.
\end{abstract}

\section{Introduction}

Until relatively recently, treatment for primary open-angle glaucoma (POAG) was restricted to medical therapy or traditional glaucoma surgeries such as drainage shunts and trabeculectomy, or laser trabeculoplasty. Trabeculectomy has a well-established IOP-lowering effect; however, it is also associated with numerous immediate and delayed postoperative complications, many of which stem from the creation of a subconjunctival bleb [1-6]. Furthermore, although it is known that suboptimal ocular outflow is a key factor in the development of glaucoma, trabeculectomy works by circumventing, rather than restoring, natural ocular outflow. In contrast, canaloplasty, a modification of viscocanalostomy, lowers IOP both safely and effectively by restoring the physiological outflow pathways $[7,8]$. Matlach et al. demonstrated that there was no significant difference in lowering medium IOP with canaloplasty compared to trabeculectomy [9].
Canaloplasty is a nonperforating, blebless technique, eliminating many of the complications frequently seen with trabeculectomy. Even those complications that are associated with the procedure are usually transient and resolve quickly [10]. This is in contrast to trabeculectomy, where blebrelated complications may occur years after the operation, thereby placing a heavy burden of postoperative care on both the patient and the physician [11]. Although canaloplasty is usually indicated for patients with POAG who have not undergone previous filtration surgery, there is evidence to suggest it may be considered in patients for whom other types of surgery have failed [12], and it may also be safely combined with cataract surgery [13].

Prior studies have reported on 1-, 2-, and 3-year results from prospective clinical trials of canaloplasty, which showed significant reductions in IOP and glaucoma medication usage in conjunction with an excellent safety profile [14-17]. The 3-year study results reported herein describe the long-term safety and efficacy of canaloplasty 
performed by a single surgeon on a large series of patients. Thus, our work intends to verify and add to the long-term outcomes of canaloplasty to further establish it as a reliably efficacious and safe modality.

\section{Patients and Methods}

2.1. Design. This paper presents the 3 -year results of a singlecenter retrospective chart review evaluating the intervention of canaloplasty at the University of Oklahoma, Dean McGee Eye Institute, performed by a single surgeon (MK) between January 2010 and December 2012. The cohort included 277 eyes affected by open-angle glaucoma. The study was performed in accordance with the principles stated in the Declaration of Helsinki. The current investigation was designed to demonstrate the safety and efficacy of canaloplasty in reducing intraocular pressure (IOP) and glaucoma medication dependence in the treatment of open-angle glaucoma (OAG). The protocol was approved by the institutional review board (IRB) at the University of Oklahoma.

All patients provided informed consent. All enrollees had a complete baseline ophthalmic examination prior to surgery that included a history of glaucoma, medication use, IOP, best-corrected visual acuity (BCVA), gonioscopy, slit lamp, and fundus examination. Postoperative follow-up examinations were at 1 day, 1 week, and 1, 3, 6, 12, 18, 24, and 36 months. All relevant information was recorded at each follow-up visit, including IOP measurements, BCVA, slit lamp examination, gonioscopy, ophthalmic medications, and adverse events. Primary endpoints included the mean IOP and mean number of glaucoma medications at each follow-up visit. Secondary endpoints included surgical/postsurgical complications. Visual acuity codes such as count fingers (CF), hand motion (HM), and light perception (LP) are converted to LogMAR following Lee et al. [18]. For a given time point, the absence of chart notes indicating hyphema (in the presence of other non-missing data collected from the same visit) was taken to indicate lack of hyphema. If the patient did not complete a visit at the given time point, the data were treated as missing.

2.2. Patient Selection. All patients were at a minimum 18 years of age at the time of enrollment, able to provide informed consent, and were scheduled for glaucoma surgery or combined cataract and glaucoma surgery. Inclusion criteria for this study included a glaucoma diagnosis of primary open-angle glaucoma (POAG), pigmentary glaucoma, or exfoliative glaucoma. Patients with advanced glaucoma exhibiting visual field loss were not excluded from the study. Patients with baseline IOP $<16 \mathrm{mmHg}$ were not excluded from the study, and none of the patients had the utilization of antifibrotics. Patients with more than two laser trabeculoplasty (LTP) procedures were excluded. Other exclusion criteria included neovascular disease, uveitis, chronic angle closure glaucoma, angle recession, and developmental or secondary glaucoma. The protocol allowed for previous surgeries that would not interfere with complete circumferential catheterization of Schlemm's canal.
TABLE 1: Study group demographics of all eyes.

\begin{tabular}{lc}
\hline Variable & Descriptive summary \\
\hline Total eyes & 277 \\
Age (years) & \\
Mean \pm SD & $72.8 \pm 10.9$ \\
Range & $40-100$ \\
Sex: count (\%) & \\
Female & $151(54.5)$ \\
Male & $126(45.5)$ \\
Race: count (\%) & \\
Asian & $1(0.4)$ \\
African American & $39(15.0)$ \\
Caucasian & $188(72.3)$ \\
Hispanic & $7(2.7)$ \\
Native American & $25(9.6)$ \\
Missing & 17 \\
Eye treated: count (\%) & \\
Right eye & $142(51.3)$ \\
Left eye & $135(48.7)$ \\
Combined cataract procedure: count (\%) & \\
Yes (phacocanaloplasty) & $127(45.8)$ \\
No (canaloplasty only) & $150(54.2)$ \\
\hline
\end{tabular}

2.3. Surgical Technique. Canaloplasty was performed using an iTrack $^{\mathrm{TM}}$ microcatheter to circumferentially viscodilate and intubate Schlemm's canal with a tensioning suture, as previously described [19].

2.4. Statistical Analysis. The primary endpoints included the IOP and number of antiglaucoma medications at 1 day, 1 week, and 1, 3, 6, 12, 18, 24, and 36 months. The secondary endpoints included visual acuity and surgical/postsurgical complications. Descriptive statistics (mean, SD, count, percent, etc.) were used to summarize the data. Comparisons of IOP, number of medications, and visual acuity were made against baseline at each time point via paired $t$-tests or Wilcoxon's signed-rank tests, as appropriate. Two-tailed $p$ values less than 0.05 were considered statistically significant. SAS (version 9.4, SAS Institute) was used for the analysis of the data.

\section{Results}

3.1. Demographics. The study cohort consisted of 277 eyes with a mean age of $72.8 \pm 10.9$ years who met inclusion and exclusion criteria, provided consent for long-term followup, and completed baseline visits. Table 1 shows the patients' demographics. Patients were predominantly white and female, with the majority $(92.1 \%)$ of the cohort diagnosed with POAG.

3.2. Change in Intraocular Pressure and Antiglaucoma Medication Use. Table 2 shows the efficacy results for all enrolled eyes in terms of IOP reduction and the number of glaucoma medications used. The IOP was significantly 
TABLE 2: Outcome measurements by visit (all eyes).

\begin{tabular}{|c|c|c|c|c|c|c|c|c|c|c|}
\hline \multirow{2}{*}{ Visit } & \multicolumn{3}{|c|}{ IOP (mmHg) } & \multicolumn{3}{|c|}{ Meds (number) } & \multicolumn{4}{|c|}{ Visual acuity $^{1}$} \\
\hline & Mean \pm SD & $N$ & $p$ value & Mean \pm SD & $N$ & $p$ value & Mean \pm SD & Snellen & $N$ & $p$ value \\
\hline Pre-op & $19.7 \pm 6.7$ & 277 & & $2.1 \pm 1.2$ & 277 & & $0.31 \pm 0.35$ & $20 / 41.1$ & 277 & \\
\hline Day 1 & $10.2 \pm 6.0$ & 271 & $<0.001$ & $0.0 \pm 0.1$ & 277 & $<0.001$ & $0.85 \pm 0.53$ & $20 / 140.2$ & 272 & $<0.0001$ \\
\hline Wk 1 & $15.3 \pm 7.1$ & 271 & $<0.001$ & $0.0 \pm 0.2$ & 276 & $<0.001$ & $0.57 \pm 0.42$ & $20 / 74.8$ & 276 & $<0.0001$ \\
\hline Mo 1 & $13.9 \pm 6.1$ & 267 & $<0.001$ & $0.1 \pm 0.4$ & 274 & $<0.001$ & $0.40 \pm 0.35$ & $20 / 49.8$ & 275 & $<0.0001$ \\
\hline Mo 3 & $14.4 \pm 5.4$ & 234 & $<0.001$ & $0.1 \pm 0.5$ & 228 & $<0.001$ & $0.29 \pm 0.34$ & $20 / 39.4$ & 239 & 0.47 \\
\hline Mo 6 & $14.6 \pm 5.1$ & 198 & $<0.001$ & $0.2 \pm 0.6$ & 205 & $<0.001$ & $0.25 \pm 0.37$ & $20 / 35.4$ & 202 & 0.07 \\
\hline Mo 12 & $14.3 \pm 4.6$ & 181 & $<0.001$ & $0.4 \pm 0.8$ & 186 & $<0.001$ & $0.24 \pm 0.40$ & $20 / 34.8$ & 185 & 0.04 \\
\hline Mo 18 & $15.0 \pm 4.6$ & 167 & $<0.001$ & $0.6 \pm 0.8$ & 144 & $<0.001$ & $0.23 \pm 0.36$ & $20 / 33.8$ & 172 & 0.06 \\
\hline Yr 2 & $14.0 \pm 4.2$ & 144 & $<0.001$ & $0.5 \pm 0.9$ & 159 & $<0.001$ & $0.22 \pm 0.29$ & $20 / 33.3$ & 143 & $<0.001$ \\
\hline Yr 3 & $15.2 \pm 4.3$ & 66 & $<0.001$ & $0.6 \pm 0.9$ & 74 & $<0.001$ & $0.20 \pm 0.24$ & $20 / 31.5$ & 65 & 0.29 \\
\hline
\end{tabular}

${ }^{1}$ LogMAR unless indicated.

TABLE 3: Outcome measurements by visit (eyes with canaloplasty only).

\begin{tabular}{|c|c|c|c|c|c|c|c|c|c|c|}
\hline \multirow{2}{*}{ Visit } & \multicolumn{3}{|c|}{ IOP $(\mathrm{mmHg})$} & \multicolumn{3}{|c|}{ Meds (number) } & \multicolumn{4}{|c|}{ Visual acuity $^{1}$} \\
\hline & Mean \pm SD & $N$ & $p$ value & Mean \pm SD & $N$ & $p$ value & Mean \pm SD & Snellen & $N$ & $p$ value \\
\hline Pre-op & $21.1 \pm 7.2$ & 150 & & $2.2 \pm 1.3$ & 150 & & $0.23 \pm 0.33$ & $20 / 34.1$ & 150 & \\
\hline Day 1 & $8.0 \pm 5.0$ & 149 & $<0.001$ & $0.0 \pm 0.1$ & 150 & $<0.001$ & $0.79 \pm 0.50$ & $20 / 124.6$ & 148 & $<0.0001$ \\
\hline Wk 1 & $15.3 \pm 7.5$ & 146 & $<0.001$ & $0.0 \pm 0.1$ & 149 & $<0.001$ & $0.55 \pm 0.43$ & $20 / 71.7$ & 149 & $<0.0001$ \\
\hline Mo 1 & $15.5 \pm 5.6$ & 145 & $<0.001$ & $0.0 \pm 0.3$ & 148 & $<0.001$ & $0.40 \pm 0.39$ & $20 / 50.2$ & 149 & $<0.0001$ \\
\hline Mo 3 & $14.8 \pm 5.1$ & 127 & $<0.001$ & $0.1 \pm 0.6$ & 123 & $<0.001$ & $0.31 \pm 0.39$ & $20 / 40.7$ & 131 & $<0.001$ \\
\hline Mo 6 & $14.9 \pm 5.4$ & 105 & $<0.001$ & $0.2 \pm 0.6$ & 109 & $<0.001$ & $0.28 \pm 0.44$ & $20 / 38.1$ & 107 & 0.03 \\
\hline Mo 12 & $14.2 \pm 4.6$ & 102 & $<0.001$ & $0.5 \pm 0.9$ & 102 & $<0.001$ & $0.28 \pm 0.48$ & $20 / 37.7$ & 101 & 0.17 \\
\hline Mo 18 & $14.6 \pm 4.5$ & 89 & $<0.001$ & $0.5 \pm 0.8$ & 76 & $<0.001$ & $0.28 \pm 0.44$ & $20 / 38.0$ & 89 & 0.04 \\
\hline Yr 2 & $13.3 \pm 4.1$ & 75 & $<0.001$ & $0.5 \pm 0.8$ & 85 & $<0.001$ & $0.26 \pm 0.36$ & $20 / 36.7$ & 74 & 0.20 \\
\hline Yr 3 & $15.0 \pm 4.6$ & 39 & $<0.001$ & $0.5 \pm 0.8$ & 43 & $<0.001$ & $0.16 \pm 0.18$ & $20 / 29.2$ & 38 & 0.05 \\
\hline
\end{tabular}

${ }^{1}$ LogMAR unless indicated.

decreased at all time points compared to baseline (all $p<0.001)$. Similarly, the medication use was significantly decreased at all postoperative time points compared to baseline $(p<0.001)$. For all eyes, the mean baseline IOP of $19.7 \pm 6.7 \mathrm{mmHg}$ was reduced to $14.3 \pm 4.6 \mathrm{mmHg}$ at 12 months, $14.0 \pm 4.2 \mathrm{mmHg}$ at 24 months, and $15.2 \pm 4.3 \mathrm{mmHg}$ at 36 months $(p<0.001)$. The mean medicine use was reduced from $2.1 \pm 1.2$ before surgery to $0.4 \pm 0.8$ at 12 months, $0.5 \pm 0.9$ at 24 months, and $0.6 \pm 0.9$ at 36 months $(p<0.001)$ (Table 2).

For the 150 eyes which underwent canaloplasty alone without phacoemulsification, the mean IOP reduced from $21.1 \pm 7.2 \mathrm{mmHg}$ at baseline to $14.2 \pm 4.6 \mathrm{mmHg}$ at 12 months, $13.3 \pm 4.1 \mathrm{mmHg}$ at 24 months and $15.0 \pm 4.6 \mathrm{mmHg}$ at 36 months $(p<0.001)$ (Table 3). Medication use for the same subset was reduced from $2.2 \pm 1.3$ at baseline to 0.5 \pm 0.9 at 12 months, $0.5 \pm 0.8$ at 24 months, and $0.5 \pm 0.8$ at 36 months $(p<0.001)$ (Table 3$)$.

A statistically significant reduction in IOP was also observed in the 127 eyes undergoing phacocanaloplasty from a mean of $18.1 \pm 5.6 \mathrm{mmHg}$ at baseline to $14.4 \pm 4.6 \mathrm{mmHg}$, $14.7 \pm 4.4 \mathrm{mmHg}$, and $15.4 \pm 4.0 \mathrm{mmHg}$ at 12,24 , and
36 months, respectively ( $p<0.001$ for 12 and 24 months, $p=0.002$ for 36 months) (Table 4). Mean medication use was reduced from $2.0 \pm 1.1$ at baseline to $0.4 \pm 0.7$ at 12 months, $0.6 \pm 0.9$ at 24 months, and $0.7 \pm 1.1$ at 36 months $(p<0.001)$.

Table 5 shows the complete and qualified success rate for canaloplasty broken down by IOP level at 6 months, 12 months, 24 months, and 36 months after surgery. Using the criteria of IOP $\leq 21 \mathrm{mmHg}$, complete success (defined as no use of antiglaucoma medication) was attained in $82.5 \%$ of patients at 6 months, $67.4 \%$ at 24 months, and $57.8 \%$ at 36 months. For IOP $\leq 15 \mathrm{mmHg}$ and $\geq 25 \%$ IOP reduction compared to baseline, complete success was deemed to be achieved in $34.0 \%$ at 6 months, $36.2 \%$ at 24 months, and $26.6 \%$ at 36 months. Qualified success, defined as patients using two or fewer antiglaucoma medications and an IOP $\leq 21 \mathrm{mmHG}$, was achieved in $91.8 \%$ of patients at 6 months, $92.2 \%$ at 24 months, and $87.5 \%$ at 36 months. Similar complete and qualified success rates were also observed for eyes with canaloplasty only (no phacocanaloplasty) (Table 6) and also for the subset of eyes with phacocanaloplasty only (Table 7) at the specified follow-up time points. 
TABLE 4: Outcome measurements by visit (eyes with phacocanaloplasty).

\begin{tabular}{lcccccccccc}
\hline \multirow{2}{*}{ Visit } & \multicolumn{3}{c}{ IOP (mmHg) } & \multicolumn{4}{c}{ Meds (number) } & \multicolumn{3}{c}{ Visual acuity $^{1}$} \\
& Mean \pm SD & $N$ & $p$ value & Mean \pm SD & $N$ & $p$ value & Mean \pm SD & Snellen & $N$ & $p$ value \\
\hline Pre-op & $18.1 \pm 5.6$ & 127 & & $2.0 \pm 1.1$ & 127 & & $0.41 \pm 0.35$ & $20 / 51.3$ & 127 \\
Day 1 & $12.9 \pm 5.9$ & 122 & $<0.001$ & $0.0 \pm 0.2$ & 127 & $<0.001$ & $\mathbf{0 . 9 1} \pm \mathbf{0 . 5 6}$ & $\mathbf{2 0 / 1 6 1 . 4}$ & $\mathbf{1 2 4}$ & $<\mathbf{0 . 0 0 0 1}$ \\
Wk 1 & $15.3 \pm 6.6$ & 125 & $<0.001$ & $0.0 \pm 0.3$ & 127 & $<0.001$ & $\mathbf{0 . 5 9} \pm \mathbf{0 . 4 0}$ & $\mathbf{2 0 / 7 8 . 5}$ & $\mathbf{1 2 7}$ & $<\mathbf{0 . 0 0 0 1}$ \\
Mo 1 & $12.0 \pm 6.1$ & 122 & $<0.001$ & $0.1 \pm 0.5$ & 126 & $<0.001$ & $0.39 \pm 0.29$ & $20 / 49.4$ & 126 & 0.72 \\
Mo 3 & $13.8 \pm 5.7$ & 107 & $<0.001$ & $0.1 \pm 0.4$ & 105 & $<0.001$ & $0.28 \pm 0.28$ & $20 / 37.8$ & 108 & $<0.001$ \\
Mo 6 & $14.4 \pm 4.6$ & 93 & $<0.001$ & $0.2 \pm 0.6$ & 96 & $<0.001$ & $0.21 \pm 0.28$ & $20 / 32.6$ & 95 & $<0.0001$ \\
Mo 12 & $14.4 \pm 4.6$ & 79 & $<0.001$ & $0.4 \pm 0.7$ & 84 & $<0.001$ & $0.20 \pm 0.27$ & $20 / 31.5$ & 84 & $<0.0001$ \\
Mo 18 & $15.5 \pm 4.8$ & 78 & $<0.001$ & $0.6 \pm 0.9$ & 68 & $<0.001$ & $0.17 \pm 0.22$ & $20 / 29.9$ & 83 & $<0.0001$ \\
Yr 2 & $14.7 \pm 4.4$ & 69 & $<0.001$ & $0.6 \pm 0.9$ & 74 & $<0.001$ & $0.18 \pm 0.17$ & $20 / 30.1$ & 69 & $<0.0001$ \\
Yr 3 & $15.4 \pm 4.0$ & 27 & 0.002 & $0.7 \pm 1.1$ & 31 & $<0.001$ & $0.24 \pm 0.29$ & $20 / 35.1$ & 27 & 0.03 \\
\hline
\end{tabular}

${ }^{1}$ LogMAR unless indicated.

TABLE 5: Success rates (all eyes).

\begin{tabular}{|c|c|c|c|c|}
\hline Success type & $\begin{array}{l}6 \text { months } \\
(N=194)\end{array}$ & $\begin{array}{c}12 \text { months } \\
(N=176)\end{array}$ & $\begin{array}{c}\text { Year 2 } \\
(N=141)\end{array}$ & $\begin{array}{c}\text { Year } 3 \\
(N=64)\end{array}$ \\
\hline \multicolumn{5}{|l|}{ Complete success $^{1}$} \\
\hline$\leq 21 \mathrm{mmHg}$ & 82.5 & 69.3 & 67.4 & 57.8 \\
\hline$\leq 21 \mathrm{mmHg}$ and $\geq 25 \%$ IOP reduction ${ }^{3}$ & 40.7 & 44.9 & 40.4 & 28.1 \\
\hline$\leq 18 \mathrm{mmHg}$ & 72.2 & 63.6 & 59.6 & 53.1 \\
\hline$\leq 18 \mathrm{mmHg}$ and $\geq 25 \%$ IOP reduction ${ }^{3}$ & 39.2 & 44.3 & 38.3 & 28.1 \\
\hline$\leq 15 \mathrm{mmHg}$ & 53.6 & 51.1 & 48.9 & 46.9 \\
\hline$\leq 15 \mathrm{mmHg}$ and $\geq 25 \%$ IOP reduction ${ }^{3}$ & 34.0 & 36.9 & 36.2 & 26.6 \\
\hline \multicolumn{5}{|l|}{ Qualified success $^{2}$} \\
\hline$\leq 21 \mathrm{mmHg}$ & 91.8 & 93.2 & 92.2 & 87.5 \\
\hline$\leq 21 \mathrm{mmHg}$ and $\geq 25 \%$ IOP reduction ${ }^{3}$ & 44.3 & 54.0 & 56.7 & 35.9 \\
\hline$\leq 18 \mathrm{mmHg}$ & 78.9 & 81.3 & 82.3 & 75.0 \\
\hline$\leq 18 \mathrm{mmHg}$ and $\geq 25 \%$ IOP reduction ${ }^{3}$ & 42.3 & 51.7 & 53.9 & 34.4 \\
\hline$\leq 15 \mathrm{mmHg}$ & 57.7 & 63.1 & 68.1 & 64.1 \\
\hline$\leq 15 \mathrm{mmHg}$ and $\geq 25 \%$ IOP reduction ${ }^{3}$ & 36.6 & 43.8 & 51.1 & 32.8 \\
\hline
\end{tabular}

${ }^{1}$ No use of antiglaucoma medication. ${ }^{2}$ Use of $\leq 2$ (including 0 ) antiglaucoma medications. ${ }^{3}$ IOP reduction from baseline/pre-op measurement.

\subsection{Intraoperative and Postoperative Complications}

3.3.1. Hyphema. The percentage of eyes with hyphema at a given time point is presented in Table 8. Except at day 1, where about half of the eyes had hyphema, the rate of this complication was low at later visits. Hyphema also tended to resolve quickly. Of the 144 eyes $(52.8 \%)$ with hyphema at 1-day post-op, $99(68.8 \%)$ were confirmed resolved by week 1,139 (96.5\%) by month 1 , and 125 (86.8\%) by month 3. The drop-off in resolved hyphema at month 3 is likely due to loss to follow-up.

3.4. Visual Recovery. For all eyes, mean pre-op visual acuity (LogMAR) was $0.31 \pm 0.35$ with a mean Snellen fraction of 20/41.1. Visual acuities were found to be significantly worse at 1 day, 1 week, and 1 month following the procedure. Post-op visual acuity was found to be $0.85 \pm 0.53$ with a Snellen fraction of 20/140.2 $(p<0.0001)$ at 1 day, $0.57 \pm 0.42$ with a Snellen fraction of $20 / 74.8(p<0.0001)$ at 1 week, and $0.40 \pm 0.35$ with a Snellen fraction of $20 /$ $49.8(p<0.0001)$ at 1 month (Table 2$)$. The post-op visual acuity was significantly better starting at 12 months, where 185 eyes had a visual acuity of $0.24 \pm 0.40$ with a Snellen fraction of $20 / 34.8(p<0.05)$. At 18 months, 172 eyes had a visual acuity of $0.23 \pm 0.36$ with a Snellen fraction of 20/33.8 $(p=0.06)$. Two years after surgery, 143 eyes had a visual acuity of $0.22 \pm 0.29$ with a Snellen fraction of $20 / 33.3(p<0.001)$. At 3 years, visual acuity was not significantly better or worse, found to be $0.20 \pm 0.24$ with a Snellen fraction of 20/31.5 $(p=0.29)$ among 65 eyes (Table 2).

Mean and SD for visual acuity (LogMAR) are presented in Table 3 for eyes undergoing canaloplasty alone $(n=150)$ and in Table 4 for eyes undergoing 
TABLE 6: Success rates (eyes with canaloplasty only).

\begin{tabular}{|c|c|c|c|c|}
\hline Success type & $\begin{array}{l}6 \text { months } \\
(N=103)\end{array}$ & $\begin{array}{l}12 \text { months } \\
(N=100)\end{array}$ & $\begin{array}{c}\text { Year } 2 \\
(N=74)\end{array}$ & $\begin{array}{c}\text { Year } 3 \\
(N=37)\end{array}$ \\
\hline \multicolumn{5}{|l|}{ Complete success $^{1}$} \\
\hline$\leq 21 \mathrm{mmHg}$ & 83.5 & 70 & 70.3 & 64.9 \\
\hline$\leq 21 \mathrm{mmHg}$ and $\geq 25 \%$ IOP reduction ${ }^{3}$ & 43.7 & 48 & 48.6 & 43.2 \\
\hline$\leq 18 \mathrm{mmHg}$ & 70.9 & 64 & 62.2 & 59.5 \\
\hline$\leq 18 \mathrm{mmHg}$ and $\geq 25 \%$ IOP reduction ${ }^{3}$ & 41.7 & 47 & 47.3 & 43.2 \\
\hline$\leq 15 \mathrm{mmHg}$ & 52.4 & 51 & 54.1 & 56.8 \\
\hline$\leq 15 \mathrm{mmHg}$ and $\geq 25 \%$ IOP reduction ${ }^{3}$ & 36.9 & 40 & 44.6 & 40.5 \\
\hline \multicolumn{5}{|l|}{ Qualified success $^{2}$} \\
\hline$\leq 21 \mathrm{mmHg}$ & 92.2 & 93 & 95.9 & 94.6 \\
\hline$\leq 21 \mathrm{mmHg}$ and $\geq 25 \%$ IOP reduction ${ }^{3}$ & 48.5 & 57 & 68.9 & 48.6 \\
\hline$\leq 18 \mathrm{mmHg}$ & 77.7 & 80 & 83.8 & 78.4 \\
\hline$\leq 18 \mathrm{mmHg}$ and $\geq 25 \%$ IOP reduction ${ }^{3}$ & 45.6 & 54 & 66.2 & 45.9 \\
\hline$\leq 15 \mathrm{mmHg}$ & 56.3 & 62 & 74.3 & 67.6 \\
\hline$\leq 15 \mathrm{mmHg}$ and $\geq 25 \%$ IOP reduction ${ }^{3}$ & 39.8 & 46 & 63.5 & 43.2 \\
\hline
\end{tabular}

${ }^{1}$ No use of antiglaucoma medication. ${ }^{2}$ Use of $\leq 2$ (including 0 ) antiglaucoma medications. ${ }^{3}$ IOP reduction from baseline/pre-op measurement.

TABLe 7: Success rates (eyes with phacocanaloplasty).

\begin{tabular}{|c|c|c|c|c|}
\hline Success type & $\begin{array}{c}6 \text { months } \\
(N=91)\end{array}$ & $\begin{array}{c}12 \text { months } \\
(N=76)\end{array}$ & $\begin{array}{c}\text { Year } 2 \\
(N=67)\end{array}$ & $\begin{array}{c}\text { Year } 3 \\
(N=27)\end{array}$ \\
\hline \multicolumn{5}{|l|}{ Complete success $^{1}$} \\
\hline$\leq 21 \mathrm{mmHg}$ & 81.3 & 68.4 & 64.2 & 48.1 \\
\hline$\leq 21 \mathrm{mmHg}$ and $\geq 25 \%$ IOP reduction ${ }^{3}$ & 37.4 & 40.8 & 31.3 & 7.4 \\
\hline$\leq 18 \mathrm{mmHg}$ & 73.6 & 63.2 & 56.7 & 44.4 \\
\hline$\leq 18 \mathrm{mmHg}$ and $\geq 25 \%$ IOP reduction ${ }^{3}$ & 36.3 & 40.8 & 28.4 & 7.4 \\
\hline$\leq 15 \mathrm{mmHg}$ & 54.9 & 51.3 & 43.3 & 33.3 \\
\hline$\leq 15 \mathrm{mmHg}$ and $\geq 25 \%$ IOP reduction ${ }^{3}$ & 30.8 & 32.9 & 26.9 & 7.4 \\
\hline \multicolumn{5}{|l|}{ Qualified success $^{2}$} \\
\hline$\leq 21 \mathrm{mmHg}$ & 91.2 & 93.4 & 88.1 & 77.8 \\
\hline$\leq 21 \mathrm{mmHg}$ and $\geq 25 \%$ IOP reduction ${ }^{3}$ & 39.6 & 50.0 & 43.3 & 18.5 \\
\hline$\leq 18 \mathrm{mmHg}$ & 80.2 & 82.9 & 80.6 & 70.4 \\
\hline$\leq 18 \mathrm{mmHg}$ and $\geq 25 \%$ IOP reduction ${ }^{3}$ & 38.5 & 48.7 & 40.3 & 18.5 \\
\hline$\leq 15 \mathrm{mmHg}$ & 59.3 & 64.5 & 61.2 & 59.3 \\
\hline$\leq 15 \mathrm{mmHg}$ and $\geq 25 \%$ IOP reduction ${ }^{3}$ & 33.0 & 40.8 & 37.3 & 18.5 \\
\hline
\end{tabular}

${ }^{1}$ No use of antiglaucoma medication. ${ }^{2}$ Use of $\leq 2$ (including 0 ) antiglaucoma medications. ${ }^{3}$ IOP reduction from baseline/pre-op measurement.

Table 8: Presence of hyphema by visit (all eyes).

\begin{tabular}{lccccc}
\hline Visit & $N$ & Present & Absent & \% present & Missing \\
\hline Pre-op & 277 & 0 & 277 & 0.0 & 0 \\
Day 1 & 273 & 144 & 129 & 52.8 & 4 \\
Wk 1 & 274 & 46 & 228 & 16.8 & 3 \\
Mo 1 & 267 & 3 & 264 & 1.1 & 10 \\
Mo 3 & 234 & 0 & 234 & 0.0 & 43 \\
\hline
\end{tabular}

phacocanaloplasty $(n=127)$, along with corresponding means in Snellen fractions and paired $t$-test results (versus pre-op VA in LogMAR). Rows in bold represent significantly worse visual acuity compared to pre-op, whereas rows in italic represent improved visual acuity. Broadly speaking, visual acuity worsens significantly after surgery but may return to preoperative values by around 3-12 months postoperatively.

\section{Discussion}

IOP reduction is the mainstay of glaucoma treatment. The 3-year results reported here showed significant and sustained pressure lowering accompanied by a low incidence of postoperative complications. The reductions in IOP achieved over the long-term follow-up period are in line 
with previously reported studies in the literature and notably the landmark multicenter prospective trial carried out at 15 clinical sites in the United States, Great Britain, and Germany in 2005 [17].

This groundbreaking study of Lewis et al. included 157 eyes of 157 OAG patients with a historical pressure of $21 \mathrm{mmHg}$ or higher, with many of them on maximum tolerated medical therapy. Canaloplasty procedures were carried out on 121 eyes while 36 eyes underwent phacocanaloplasty. Of the 89 procedures performed with a successful placement of a suture, there was a $34 \%$ mean decrease in IOP from baseline $(23.5 \pm 4.5$ to $15.5 \pm 3.5 \mathrm{mmHg})$ and a $53 \%$ mean reduction in postoperative medications $(1.9 \pm 0.8$ to $0.9 \pm 0.9)$ at three-year follow-up. When phacoemulsification was combined with canaloplasty and successful suture placement, 27 eyes had a $42 \%$ mean decrease in IOP $(23.5 \pm 5.2$ to 13.6 $\pm 3.6 \mathrm{mmHg}$ ) and an $80 \%$ mean reduction of postoperative medications $(1.5 \pm 1$ to $0.3 \pm 0.5)$.

Another long-term study by Bull et al. investigated the efficacy of canoloplasty and phacocanaloplasty on European eyes with open-angle glaucoma [7]. One hundred and nine eyes were included in the study with successful intracanalicular suture tensioning occurring in 98 eyes (89.9\%), canaloplasty alone performed in 93 eyes (85.3\%) and phacocanaloplasty performed in 16 eyes (14.7\%) with 3year follow-up data available for 96 eyes (88.1\%). In the canaloplasty alone group, the mean baseline IOP of $23.0 \pm 4.3 \mathrm{mmHg}$ on $1.9 \pm 0.7$ medications was significantly reduced to $15.1 \pm 3.1 \mathrm{mmHg}$ on $0.9 \pm 0.9$ medications while the phacocanaloplasty group's mean baseline IOP of $24.3 \pm 6.0 \mathrm{mmHg}$ on $1.5 \pm 1.2$ medications was significantly reduced to $13.8 \pm 3.2 \mathrm{mmHg}$ on $0.5 \pm 0.7$ medications $(p<0.0001)$. The outcomes in several recently published studies have equaled or surpassed those of the 2005 trial. In 2011, Grieshaber et al. published the results of a prospective study of 32 patients with OAG in which the mean IOP fell from $27.3 \pm 5.6 \mathrm{mmHg}$ preoperatively to $12.8 \pm 1.5 \mathrm{mmHg}$ at 12 months and $13.1 \pm 1.2 \mathrm{mmHg}$ at 18 months [16]. A more recent study by Brusini of 214 eyes from 185 OAG patients with a maximum of four-year follow-up reported a mean IOP reduction of $42.2 \%$ [20].

Compared to these previous trials, our large-scale study of 277 eyes with successful suture tensioning demonstrated a combined $23 \%$ reduction in IOP from baseline $(19.7 \pm 6.7$ to $15.2 \pm 4.3 \mathrm{mmHg}$ ) and $71 \%$ reduction in medication use $(2.1 \pm 1.2$ to $0.6 \pm 0.9)$ at three-year follow-up. The 150 patients undergoing canaloplasty alone had a $29 \%$ reduction in IOP $(21.1 \pm 7.2$ to $15.0 \pm 4.6 \mathrm{mmHg})$ and $77 \%$ reduction in medication use postoperatively $(2.2 \pm 1.3$ to $0.5 \pm 0.8)$ at three years. The 127 eyes undergoing phacocanaloplasty had a $17 \%$ mean decrease in IOP $(18.1 \pm 5.6$ to $15.1 \pm 4.0 \mathrm{mmHg})$ and a $65 \%$ reduction in medication use postoperatively $(2.0 \pm 1.1$ to $0.7 \pm 1.1)$ at three years. These patients had a lower baseline preoperative IOP as they were predominately undergoing surgical intervention for cataract, rather than increased pressure. In all cases, our study reached a similar endpoint when compared to previous studies, with a significant reduction in both IOP and postoperative medication use. This large data set, with 277 procedures performed by a single surgeon, acts to further verify and add to the efficacy of canaloplasty as a viable option for the treatment of OAG. A recent review by Cagini et al. summarizes the potential pitfalls associated with canaloplasty, noting that the meta-analysis of the literature demonstrates a relatively low rate of complications, particularly severe ones, compared to trabeculectomy [21]. Potential intraoperative complications associated with canaloplasty include inability to cannulate Schlemm's canal, Descemet membrane detachment, and improper microcatheter passage $[7,17,18]$. In the current investigation, patients were excluded due to inability to place a suture tension. The most frequent postoperative complications associated with canaloplasty include hyphema or microhyphema, cataract formation, IOP spikes, and hypotony [14]. While transient hyphema was the most common side effect in the current study, a study by Grieshaber et al. has shown that hyphema can, in fact, be considered to be a sign of successful reconnection with the ocular venous system and therefore of good prognosis [22]. Sustained hypotony and related complications, however, did not occur. Our own study confirmed the robust safety profile of canaloplasty and replicated the low rate of intraoperative and postoperative complications reported in previous studies. The high rate of attrition at year three in this study is attributable to few postoperative complications, allowing for prompt referral back to their primary doctors.

\section{Conclusion}

This single-center clinical trial provides further evidence of the significant IOP lowering efficacy of canaloplasty with suture tensioning, with continued control through a 3-year postoperative period. The risk profile of canaloplasty was favorable and consistent with the well-documented, lower risks associated with other nonpenetrating procedures. Canaloplasty's safety profile and long-term efficacy make it a viable option for the majority of glaucoma patient types. It can be used in conjunction with existing drug-based glaucoma treatments, after laser or other types of incisional surgery, and does not preclude or affect the outcome of future surgery. It is a procedure that offers a high probability of success in effectively lowering IOP both alone and combined with phacoemulsification.

\section{Conflicts of Interest}

The authors have no financial or proprietary interest in any material or method mentioned.

\section{References}

[1] E. Jones, J. Clarke, and P. T. Khaw, "Recent advances in trabeculectomy technique," Current Opinion in Ophthalmology, vol. 16, pp. 107-113, 2005.

[2] N. S. C. Borisuth, B. Phillips, and T. Krupin, "The risk profile of glaucoma filtration surgery," Current Opinion in Ophthalmology, vol. 10, pp. 112-116, 1999.

[3] S. J. Gedde, L. W. Herndon, J. D. Brandt, D. L. Budenz, W. J. Feuer, and J. C. Schiffman, "Surgical complications in the Tube Versus Trabeculectomy Study during the first year of follow- 
up; the Tube Versus Trabeculectomy Study Group," American Journal of Ophthalmology, vol. 143, pp. 23-31, 2007.

[4] I. U. Scott, D. S. Greenfield, J. Schiffman et al., "Outcomes of primary trabeculectomy with the use of adjunctive mitomycin," Archives of Ophthalmology, vol. 116, pp. 286-291, 1998.

[5] H. D. Jampel, D. C. Musch, B. W. Gillespie et al., "Perioperative complications of trabeculectomy in the collaborative initial glaucoma treatment study (CIGTS)," American Journal of Ophthalmology, vol. 140, pp. 16-22, 2005.

[6] B. Edmunds, J. R. Thompson, J. F. Salmon, and R. P. Wormald, "The national survey of trabeculectomy. III. Early and late complications," Eye, vol. 16, pp. 297-303, 2002.

[7] H. Bull, K. von Wolff, N. Körber, and M. Tetz, "Three-year canaloplasty outcomes for the treatment of open-angle glaucoma: European study results," Graefe's Archive for Clinical and Experimental Ophthalmology, vol. 249, no. 10, pp. 1537$1545,2011$.

[8] M. C. Grieshaber, A. Pienaar, J. Olivier, and R. Stegmann, "Canaloplasty for primary open-angle glaucoma: long-term outcome," The British Journal of Ophthalmology, vol. 94, no. 11, pp. 1478-1482, 2010.

[9] J. Matlach, C. Dhillon, J. Hain, G. Schlunck, F. Grehn, and T. Klink, "Trabeculectomy versus canaloplasty (TVC study) in the treatment of patients with open-angle glaucoma: a prospective randomized clinical trial," Acta Ophthalmologica, vol. 93, pp. 753-761, 2015.

[10] T. Klink, E. Panidou, B. Kanzow-Terai, J. Klink, G. Schlunck, and F. J. Grehn, "Are there filtering blebs after canaloplasty?," Journal of Glaucoma, vol. 21, no. 2, pp. 89-94, 2012.

[11] S. Zahid, D. C. Musch, L. M. Niziol, and P. R. Lichter, "Collaborative initial glaucoma treatment study groupRisk of endophthalmitis and other long-term complications of trabeculectomy in the collaborative initial glaucoma treatment study (CIGTS)," American Journal of Ophthalmology, vol. 155, no. 4, pp. 674-680.e1, 2013.

[12] P. Brusini and C. Tosconi, "Canaloplasty after failed trabeculectomy: a possible option," Journal of Glaucoma, vol. 23, no. 1, pp. 33-34, 2014.

[13] M. Tetz, H. Koerber, B. J. Shingleton et al., "Phacoemulsification and intraocular lens implantation before, during, or after canaloplasty in eyes with open-angle glaucoma: 3-year results," Journal of Glaucoma, vol. 24, no. 3, pp. 187-194, 2015.

[14] R. A. Lewis, K. von Wolff, M. Tetz et al., "Canaloplasty: circumferential viscodilation and tensioning of Schlemm's canal using a flexible microcatheter for the treatment of open-angle glaucoma in adults: interim clinical study analysis," Journal of Cataract and Refractive Surgery, vol. 33, pp. 1217-1226, 2007.

[15] R. A. Lewis, K. von Wolff, M. Tetz et al., "Canaloplasty: circumferential viscodilation and tensioning of Schlemm canal using a flexible microcatheter for the treatment of open-angle glaucoma in adults: two-year interim clinical study results," Journal of Cataract and Refractive Surgery, vol. 35, pp. 814824, 2009.

[16] M. C. Grieshaber, S. Fraenk, A. Schoetzau, J. Flammer, and S. Orgul, "Circumferential viscocanalostomy and suture canal distension (canaloplasty) for Whites with open-angle glaucoma," Journal of Glaucoma, vol. 20, pp. 298-302, 2011.

[17] R. A. Lewis, K. von Wolff, M. Tetz et al., "Canaloplasty: threeyear results of circumferential viscodilation and tensioning of Schlemm canal using a microcatheter to treat open-angle glaucoma," Journal of Cataract \& Refractive Surgery, vol. 37, pp. 682-690, 2011.

[18] J. W. Lee, J. S. Lai, D. W. Yick, and R. K. Tse, "Retrospective case series on the long-term visual and intraocular pressure outcomes of phacomorphic glaucoma," Eye, vol. 24, no. 11, pp. 1675-1680, 2010.

[19] M. A. Khaimi, "Canaloplasty: a minimally invasive and maximally effective glaucoma treatment," Journal of Ophthalmology, vol. 2015, Article ID 485065, 5 pages, 2015.

[20] P. Brusini, "Canaloplasty in open-angle glaucoma surgery: a four-year follow-up," The Scientific World Journal, vol. 2014, Article ID 469609, 7 pages, 2014.

[21] C. Cagini, C. Peruzzi, T. Fiore, L. Spadea, M. Lippera, and S. Lippera, "Canaloplasty: current value in the management of glaucoma," Journal of Ophthalmology, vol. 2016, Article ID 7080475, 6 pages, 2016.

[22] M. C. Grieshaber, A. Schoetzau, J. Flammer, and S. Orgül, "Postoperative microhyphema as a positive prognostic indicator in canaloplasty," Acta Ophthalmologica, vol. 91, no. 2, pp. 151-156, 2013. 


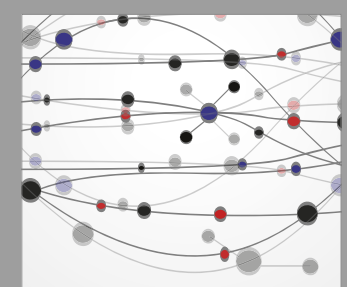

The Scientific World Journal
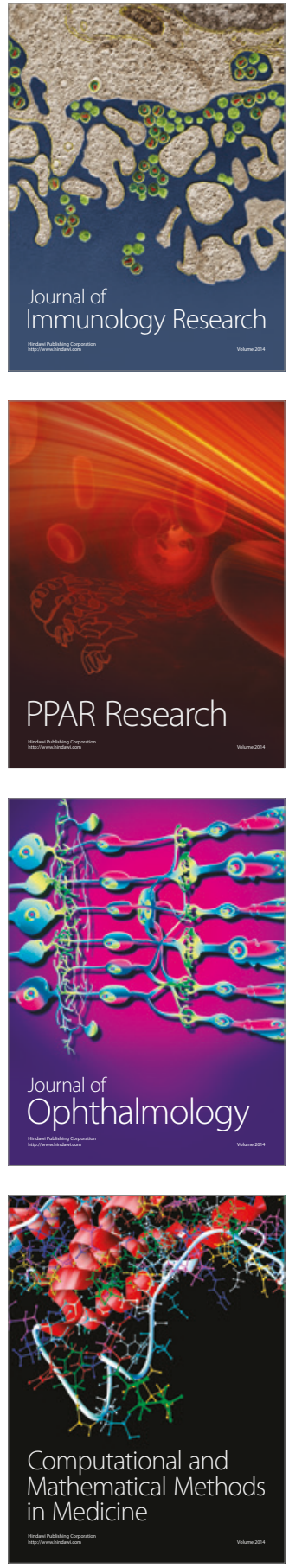

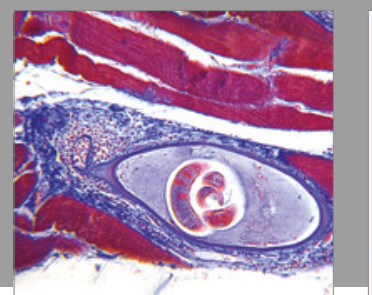

Gastroenterology Research and Practice
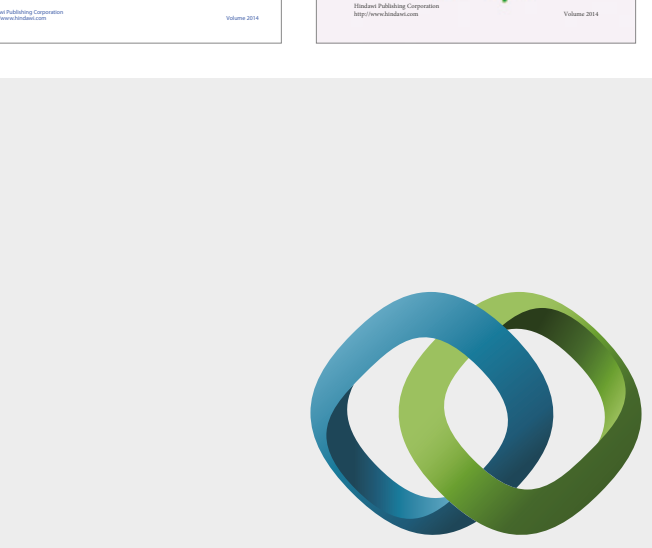

\section{Hindawi}

Submit your manuscripts at

https://www.hindawi.com
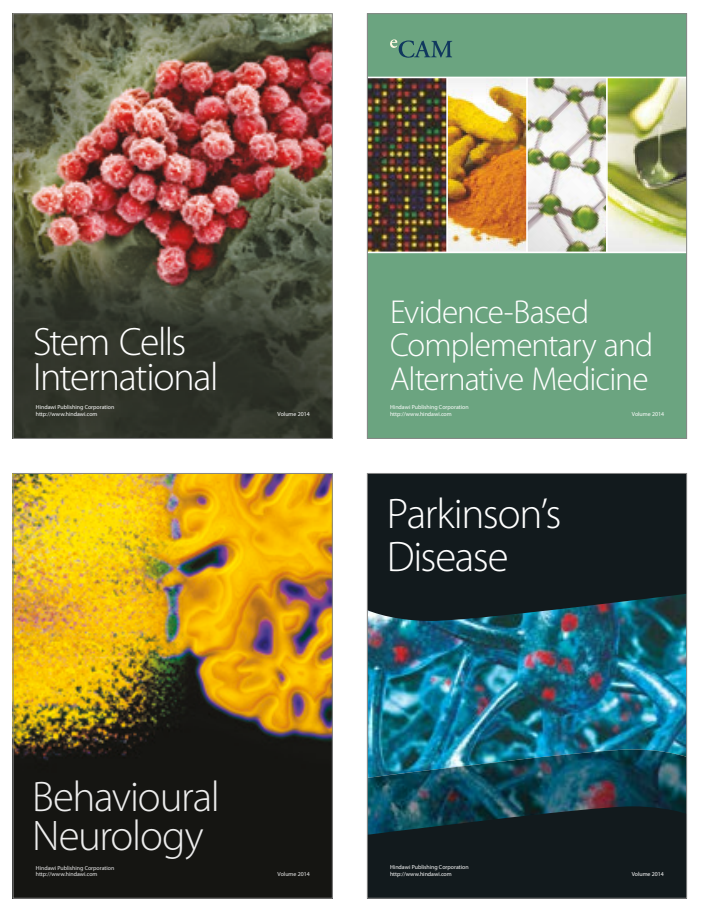
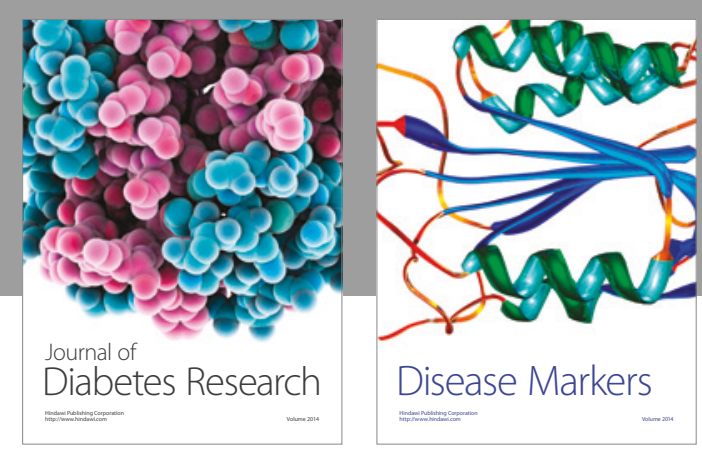

Disease Markers
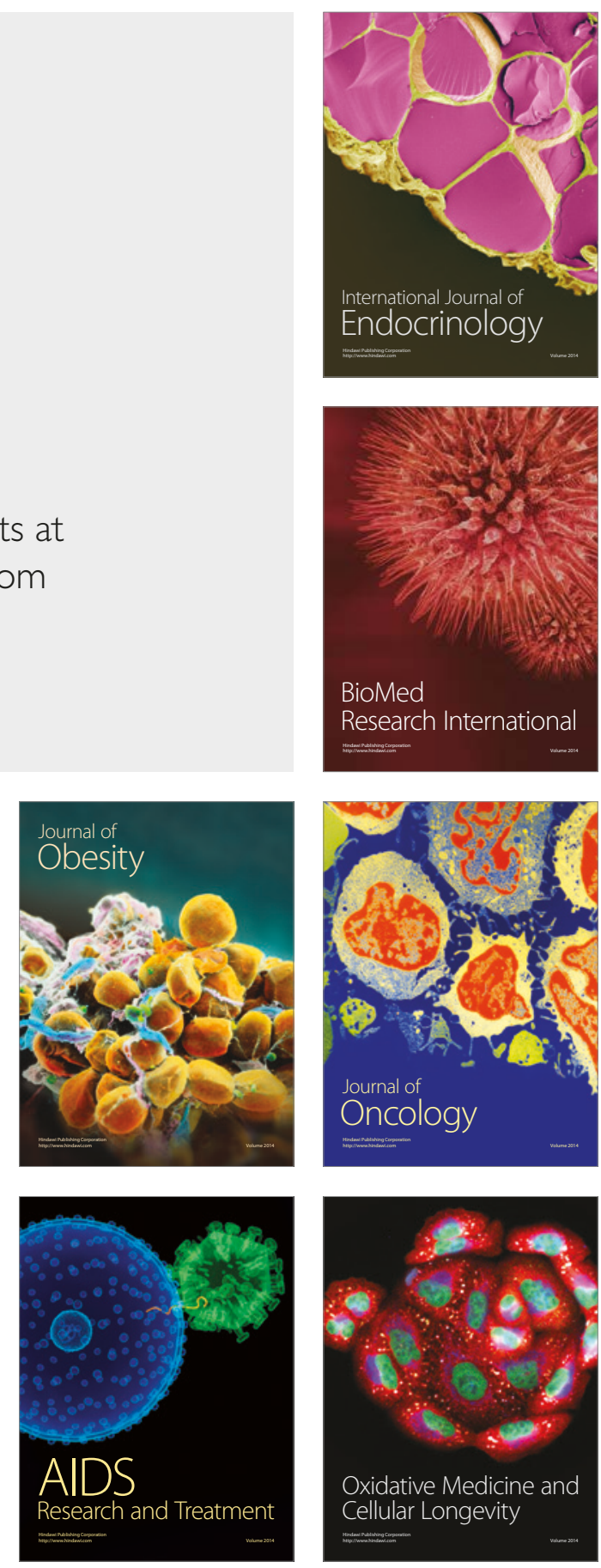